\title{
Novel Approaches for Minutiae Verification in Fingerprint Images
}

\author{
Sharat Chikkerur, Venu Govindaraju \\ Center for Unified Biometrics and Sensors \\ University at Buffalo, NY \\ $\{$ ssc5,govind $\} @$ buffalo.edu
}

\author{
Sharath Pankanti, Ruud Bolle, Nalini Ratha \\ IBM T. J. Watson Research Center \\ Hawthorne, NY \\ $\{$ sharat,bolle,ratha\}@us.ibm.com
}

\begin{abstract}
A majority of the existing fingerprint recognition algorithms are based on matching minutia features. Therefore, minutiae extraction is one of the critical steps in fingerprint verification algorithms. Poor quality fingerprint images lead to missing and spurious minutiae that degrate the performance of the matching system. We propose two new techniques for minutiae verification based on non-trivial gray level features. The proposed features intuitively represents the structual properties of the minutiae neighborhood leading to better classification. We use directionally selective steerable wedge filters to differentiate between minutiae and non-minutiae neighborhoods We also propose a second technique based on Gabor expansion that results in even better discrimination. We present an objective evaluation of both the algorithms.
\end{abstract}

\section{Introduction}

Fingerprint verification has emerged as one of the most reliable means of biometric authentication due to its universality,distinctiveness, permanence and accuracy [10]. Most of the existing fingerprint recognition algorithms are based on matching minutiae features. Minutiae represent local deviation in the flow of ridges .Given a fingerprint image, all the minutiae locations, orientations and structural relationship among the points are determined and stored as part of the template. During matching, the deformation between two such point sets is recovered and the point correspondences are determined to generate a similarity score [10]. Despite of extensive research efforts, reliable matching of fingerprints still remains a challenging problem. No two different impressions of the fingerprint are identical even when they come from the same individual. The matching algorithm has to be therefore invariant to changes in orientation, displacement,occlusion and missing features. Varying skin conditions may lead to poor quality images that can lead to feature extraction errors and subsequently error during recognition.

\subsection{Problem Description}

Many of the minutiae extraction algorithms are sequential in nature, resulting in error propagation in each of the stages. Error resulting from poor quality images can be eliminated using enhancement techniques like Gabor filtering [6]. Variations due to displacement and elastic deformation are handled by the matching algorithm itself. In this paper, we deal with errors introduced during feature extraction. The feature extraction results in the following type of errors

1. Missing minutiae: The feature extraction algorithm fails to detect existing minutia when the minutiae is obscured by surrounding noise or poor ridge structures.

2. Spurious minutia: The feature extraction algorithm falsely identifies a noisy ridge structure such as a crease, ridge break or gaps as minutiae.

When the feature extraction is performed using binarization and thinning, spurs, bridges, opposing minutiae, triangles, ladders are some of the structures leading to false minutiae detection [10]. Gray level based feature extraction methods such as the ridge following approach proposed by Maio and Maltoni [9] can eliminate many of the sources of error that are caused by binarization and thinning. However, in poor contrast or poor quality images where the local maxima of the ridges cannot be reliably located, false positives are still introduced. Therefore, minutiae extraction is usually followed by a post-processing step that tries to eliminate false positives. It has been shown that this refinement can result in considerable improvement in the accuracy of a minutia based matching algorithm [12].

In this paper we present two approaches to eliminate false minutiae that are invariant to feature extraction process.Minutiae represent local deviation in the flow of ridges. Although many different types of fingerprint features have been identified, ridge endings and bifurcation account for a 


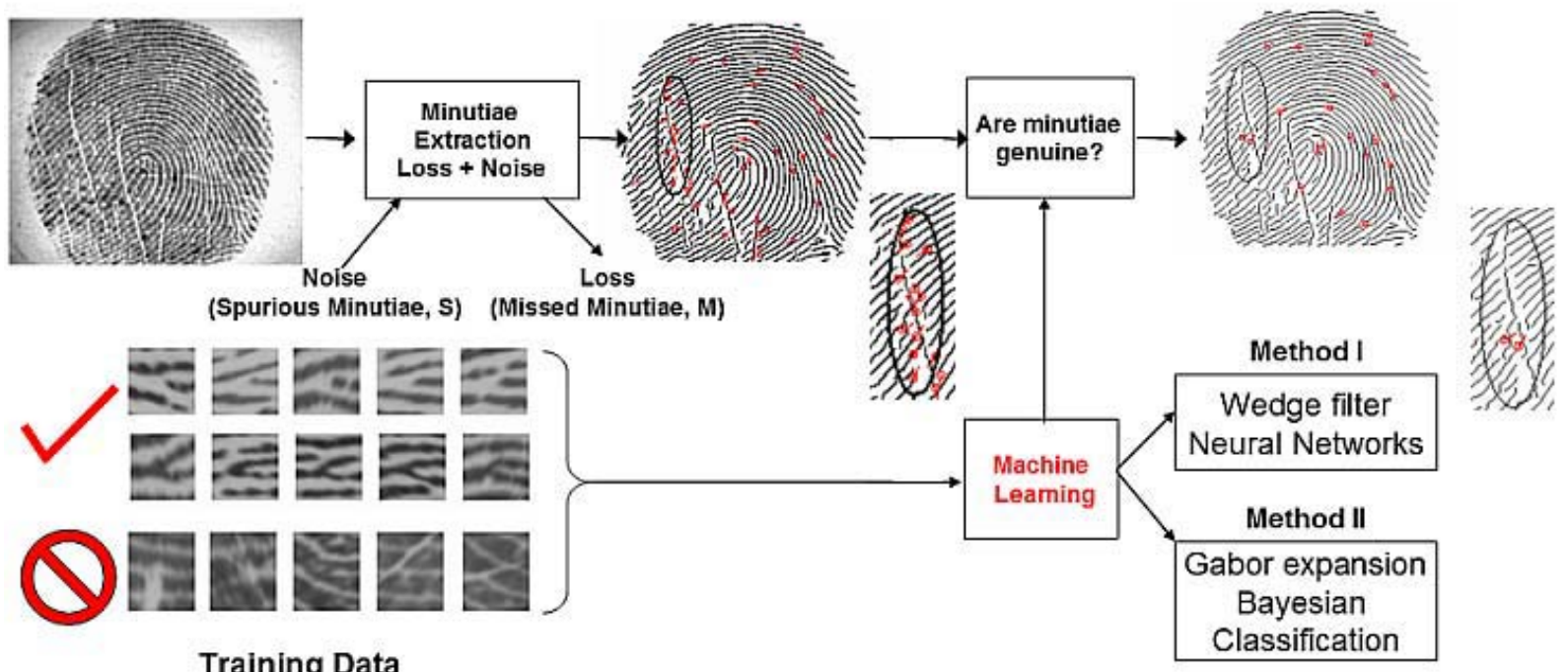

Figure 1. Overview of the proposed approach

majority of those features. We treat both ridge endings and bifurcation uniformly since their type can get exchanged with slight variation of pressure. Also, the majority of the existing fingerprint matching algorithms do not distinguish between the minutia types during verification.

\subsection{Prior related work}

Existing methods to filter spurious minutiae can be categorized into two methods

1. Structural post processing: Structural post processing methods prune spurious minutia based on heuristics rules that use the relative location of the detected minutia, length of the ridges and other structural information. However, these rules are very specific to the feature extraction algorithm. Xiao and Raafat [18] provided taxonomy of structures resulting from thinning that lead to spurious minutia and proposed heuristic rules to eliminate them. Hung [7] proposed a graphbased algorithm that exploits the duality of the ridges and bifurcation. The binarization and thinning is carried on positive and negative gray level images resulting in ridge skeleton and its complementary valley skeleton. Minutiae features that occur in both images are retained while eliminating the false positivies.

2. Gray level image based filtering:Gray scale based techniques use the gray scale values in the immediate neighborhood of the candidate minutiae to validate them. Prabhakar et. al [12] proposed direct pixel value based approach to eliminate false minutiae. A $64 \times 64$ region of the neighborhood surrounding the minutiae is normalized w.r.t orientation and brightness variation.
Horizonatally oriented Gabor filters are used to enhance the region and the pixels in the central $32 \times 32$ regions is used as features to distinguish between minutiae and non-minutiae neighborhoods. The classification is done using a Learning Vector Quantizer that uses the central 1024 pixels as input and classifies the region as ridge, bifurcation or non-minutiae. Maio and Maltoni [2] proposed a neural network based approach for minutiae filtering. In this method, the minutiae neighborhood is normalized w.r.t ridge frequency in addition to orientation and brightness variations. The dimensionality of the feature set is reduced by using Karhunen Leove Transform. Classification is done using a shared weights neural network that uses both positive and negative images of the minutiae neighborhood to exploit the duality of the ridge and bifurcation. Although this approach leads to significant reduction in the number of false positivies and exchanged minutiae, it also increases the number of missing minutiae. Both the approaches also differ on their definition of non-minutiae neighborhoods. While Prabhakar et al. [12] treat even plain ridges as non-minutiae, Maio and Maltoni additionally use the actual false positives given by their feature extraction algorithm [9] as nonminutiae. Recently, Wu et al. [17] developed an optimal filter based approach to detection of creases in fingerprint images. In this approach, the ridge neighborhood containing the crease is represented using a parameterized rectangle. They employ a multiple channel filtering framework to detect creases in different orientations. However, this method cannot detect spurious minutiae that occur in smudged or exceptionally dry parts of the fingerprint. 


\subsection{Overview}

Figure 1 shows an overview of the proposed approach. Firstly, we generate training samples consisting of minutiae and non-minutiae neighborhoods. Then, given sufficient labelled data, the system uses a suitable feature set and a machine learning algorithm to 'learn' the difference between genuine minutiae and non-minutiae neighborhoods. We propose two feature descriptors that can be used to differentiate between minutiae and non minutiae neighborhoods. The first approach is based on the response of the minutiae neighborhood to a bank of steerable wedge filters. The response is fed to a feedforward back propagation network to classify the inputs as either minutiae or non minutiae neighborhood. The second and also the more accurate approach is based expressing the minutiae neighborhoods as a linear sum of basis images made up of multi-resolution Gabor elementary functions. We utilize a Bayesian classification for this purpose. The particular choice of classifiers is explained in subsequent sections.

The rest of the paper is organized as follows. Section 2 explains the use of wedge filter for minutiae verification. The second approach using Gabor expansion is presented in section 3. Experimental verification and objective evaluation of the techniques is presented in section 4. Finally conclusion and future work are discussed in section 5 .

\section{Steerable Wedge Filters}

Steerable filters have been used for some time to analyze local orientation in images. Steerable filters allow us to compute the responses at different orientations as a linear combination of responses to a set of basis filters. Freeman and Aldeson [5] first developed the concept of steerable filters using directional derivatives of Gaussians. However, the symmetry of these derivatives imposes an angular periodicity of $\pi$ on these filter responses irrespective of the underlying image structure. The bimodal response is not very useful in junction analysis and pattern recognition application where a full range resolution $([0,2 \pi])$ is needed. Simoncelli and Farid [16] propose asymmetrical filters that exhibit a unimodal response. A brief overview of these filters is provided for completeness. Further details are provided in [5] and [16]. Consider a bank of two filters given by

$$
\begin{array}{r}
G_{n}^{0}(r, \theta)=\cos (\theta) \frac{\partial g^{n}(r)}{\partial r^{n}}, G_{n}^{\pi / 2}(r, \theta)=\sin (\theta) \frac{\partial g^{n}(r)}{\partial r^{n}} \\
g(r)=e^{\frac{-r^{2}}{2}}
\end{array}
$$

The subscript indicates the order of the derivative while the superscript indicates the orientation of the basis filters. It can be easily shown that using these two basis filters, a filter oriented in an arbritrary direction $\phi$ can be constructed

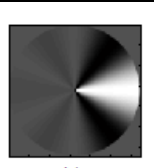

(a)

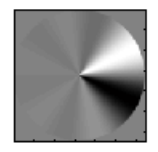

(f)

(g)

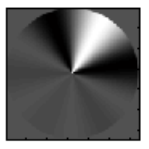

(b)

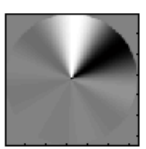

(g)

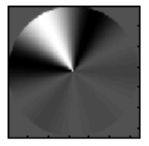

(c)

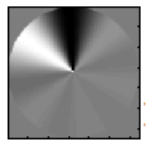

(h)

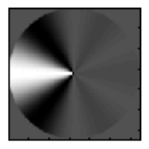

(d)

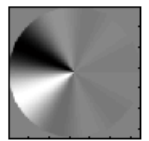

(i)

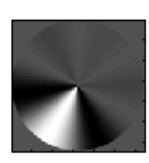

(e)

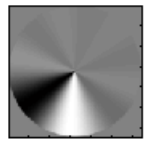

(j)
Figure 2. Bank of steerable wedge filters for $\mathrm{N}=5$.(a-e) evenly symmetric filters, (f)-j)oddly symmetric filters

using

$$
h(\phi)=G_{n}^{0} \cos (\phi)+G_{n}^{\pi / 2} \sin (\phi)
$$

$\cos (\phi)$ and $\sin (\phi)$ form the interpolation function. Simoncelli et. al [16] further impose a hilbert transform relation between the even and odd components of the filter. The filters are obtained using

$$
h_{e}(\phi)=\sum_{n=1}^{N} \omega_{n} \cos (n \phi), h_{o}(\phi)=\sum_{n=1}^{N} \omega_{n} \sin (n \phi)
$$

The weights $\omega_{n}$ are chosen so as to localize the energy response. To construct a filter in arbitrary orientation, it is expressed as a linear combination of basis filters $h\left(\phi-\alpha_{n}\right)$.

$$
f^{\alpha}(r, \phi)=h(\phi-\alpha) g(r)=g(r) \sum_{n=1}^{\hat{N}} k_{n}(\alpha) h\left(\phi-\alpha_{n}\right)
$$

$\mathrm{g}(\mathrm{r})$ represents an arbitrary radial function with compact support. $k_{n}(\alpha)$ represents the interpolation function and $\alpha_{n}$ represents the orientations of the basis filters. Figure 2 shows the basis filters for $\mathrm{N}=5$.

\subsection{Minutiae Verification}

Minutiae is marked by local deviation in the ridge flow, with three dominant local directions corresponding to the two branches of the bifurcation and the parallel ridge direction. Plain ridges are marked by a single dominant direction aligned with the horizontal. On the other hand, false positives encountered around the creases and noisy regions of the fingerprint are marked by random and multiple dominant directions. Creases, in particular are identified by dominant components in directions orthogonal to the ridge flow.Figure 3 shows response of a wedge filter to a prototypical bifurcation and non minutiae region. The distinction between the two regions is clearly evident. 

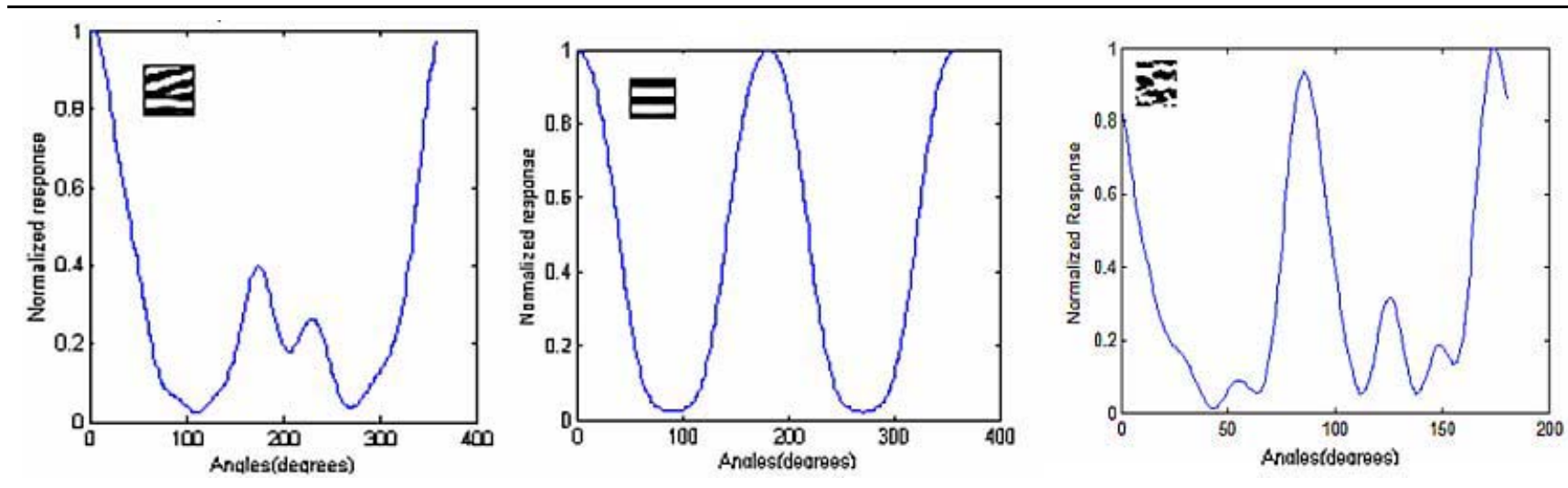

Figure 3. Response of the wedge filter to a prototypical bifurcation and plain ridge

Minutiae verification using wedge filter consists of the following steps (i) A $24 \times 24$ region surrounding each potential minutiae is normalized w.r.t orientation. (ii) The local block is enhanced using the algorithm described in [1]. This makes it invariant to brightness and contrast variation. (iii) The orientation response of the block is computed in the interval $[-\pi, \pi]$ using $\mathrm{N}=18$ basis filters. (iv) The responses are then classified into minutiae and non-minutiae region using a neural network classifier.

\subsection{Neural Networks}

An analysis using Fisher discriminant analysis [4] shows us that the two classes are not linearly separable. Therefore it requires a more sophisticated classification step. It is well known that a multi-layered feed forward neural network is capable of classifying non-linearly separated data. We use a three layered , 180-10-1 neuron architecture for our purpose. We use resilient back propagation [15] algorithm to train the network. The resilient back propagation algorithm works by considering only the sign of the derivative for updating the weights. The change in weight is determined by a separate parameter. Therefore unlike gradient descent methods, the rate of convergence does not slow down as the slope of the error gradient decreases with time. The evaluation of the classifiers is given in sectionsec:evaluation

\section{Gabor expansion}

Gabor elementary functions have been previously used for enhancement of fingerprint images [6] and also for synthetic fingerprint generation [14]. They have important signal properties such as optimal joint space frequency resolution [13]. Daugman [3] and Lee [8] have used Gabor elementary functions as basis functions to represent generic 2D images.Gabor elementary functions form a very intuitive representation of fingerprint images since they capture the periodic,yet non-stationary nature of the fingerprint regions. However, unlike fourier bases or discrete cosine bases, using Gabor elementary functions have the following problems. (i)From a signal processing point of view, they do not form a tight frame. This means that the image cannot be represented as a linear superposition of the Gabor elementary functions with coefficients derived by projecting the image onto the same set of basis functions. However, Lee [8] has derived conditions under which a set of self similar Gabor basis functions form a complete and approximately orthonormal set of basis functions. (ii) They are biorthogonal bases. This means that the basis functions used to derive the coefficients(analysis functions) and the basis functions used to reconstruct the image (synthesis functions) are not identical or orthogonal to each other.However, Daugman proposes a simple optimization approach to obtain the coefficients.

Daugman gives the following form for the 2D Gabor elementary function

$$
\begin{array}{r}
G(x, y)=\exp \left(-\pi\left[\left(x-x_{0}\right)^{2} \alpha^{2}+\left(y-y_{0}\right)^{2} \beta^{2}\right]\right) . \\
\exp \left(-2 \pi i\left[u_{0}\left(x-x_{0}\right)+v_{0}\left(y-y_{0}\right)\right]\right)
\end{array}
$$

$x_{0}$ and $y_{0}$ represent the center of the elementary function in the spatial domain. $u_{0}$ and $v_{0}$ represent the modulation frequencies. $\alpha^{2}$ and $\beta^{2}$ represent the variance along the major and minor axes respectively and therefore the extent of support in the spatial domain.

\subsection{Feature representation}

We use a multi-resolution representation using Gabor expansion to distinguish between minutiae and non minutiae neighborhood.The bases are derived using a self similar Gabor elementary functions computed at multiple scales and orientations. Figure 4 displays some of the basis functions used to derive the feature representation. The basis function 


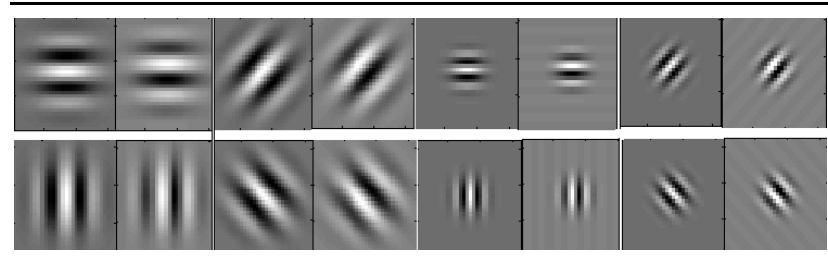

Figure 4. Some of the Gabor basis functions used to represent the minutiae neighborhoods

at scale $\mathrm{m}$, displacement $(\mathrm{p}, \mathrm{q}) \in\left(0,2^{m}, 2^{2 m} \ldots, N\right)$ and orientation $\theta$ is given by [3]

$$
\begin{aligned}
\Psi_{m p q \theta}(x, y) & =2^{-m} G\left(x^{\prime}, y^{\prime}\right) \\
x^{\prime} & =2^{-m}[x \cos (\theta)+y \sin (\theta)]-p \\
y^{\prime} & =2^{-m}[-x \sin (\theta)+y \cos (\theta)]-q
\end{aligned}
$$

We represent a $32 \times 32$ region around the fingerprint image using basis functions that span two scales and four orientations resulting in 272 such basis functions. The image can therefore approximately represented using

$$
I(x, y)=\sum_{n=1}^{272} a_{n} G_{n}(x, y)
$$

The optimal coefficients are obtained a gradient descent approach as suggested by Daugman in [3]. At each iteration the coefficient is incremented by an amount

$\Delta a_{n}=G_{n}(x, y) \cdot I(x, y)-G_{n}(x, y) \cdot\left(\sum_{k} G_{k}(x, y) I(x, y)\right)$

Figure 5 shows examples of normalized minutiae neighborhoods and their approximate reconstruction using Gabor expansion.

\subsection{Bayesian Classification}

We use a deterministic Bayesian classifier [4] in order to distinguish between minutiae and non-minutiae neighborhoods. We can treat minutiae and non minutiae as two states of nature $\omega_{1}$ and $\omega_{2}$. The coefficients can be represented using a feature vector $\vec{x}$. We decide the given sample as belonging to $\omega_{1}$ if the posterior probability $p\left(\omega_{1} \mid \vec{x}\right)$ is greater than $p\left(\omega_{2} \mid \vec{x}\right)$. According to Baye's rule, the posterior probabilities can be calculated using

$$
p\left(\omega_{i} \mid \vec{x}\right)=\frac{p\left(\vec{x} \mid \omega_{i}\right) p\left(\omega_{i}\right)}{\sum_{i} p\left(\vec{x} \mid \omega_{i}\right)}
$$

In practice, we usually take a decision that maximizes the $\log$ probabilities $\ln \left(p\left(\omega_{i} \mid \vec{x}\right)\right)$. In terms of discriminant functions, we decide that the feature belongs to $\omega_{1}$ if $g_{1}(\vec{x})>$

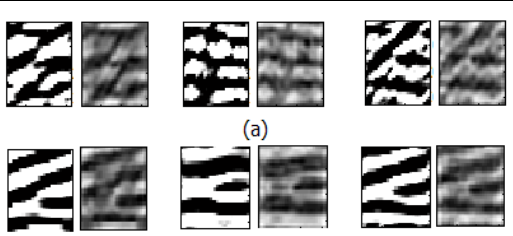

(b)

Figure 5. Neighborhoods reconstructed using Gabor functions

$g_{2}(\vec{x})$ where $g_{i}(\vec{x})=\ln p\left(\vec{x} \mid \omega_{i}\right)+\ln p\left(\omega_{i}\right)$. Also, we have observed that all conditional probabilities, $p\left(\vec{x} \mid \omega_{i}\right)$ can be represented as univariate gaussian. We further assume that the features are independent of each other. The discriminant function can therefore be expressed as

$$
g_{i}(\vec{x})=-\frac{1}{2}\left(\vec{x}-\overrightarrow{\mu_{i}}\right)^{t} \Sigma_{i}^{-1}\left(\vec{x}-\overrightarrow{\mu_{i}}\right)-\frac{1}{2} \ln \left|\Sigma_{i}\right|
$$

Here $\overrightarrow{\mu_{i}}$ represents the mean vector for the class $\omega_{i}$ and $\Sigma_{i}$ consists of a diagonal covariance matrix since we assume each feature to be independent of each other.

\subsection{Feature Selection}

We notice that all the 272 features do not sufficiently discriminate the two classes $\omega_{1}$ and $\omega_{2}$. We therefore consider only those features that sufficiently disciriminate among the classes. We define the discriminability metric $D$ of each feature $x_{i}$ as

$$
D_{i}=\frac{\left|\mu_{1 i}-\mu_{2 i}\right|}{\sigma_{1 i}+\sigma_{2 i}}
$$

We select only those features where $D_{i}>0.5$. This results in retaining 12 of the 272 total features. Only these features are used for the Bayesian classification.

\section{Experimental Verification}

\subsection{Wedge filter approach}

4.1.1. Test data The test data consisted of 2000 genuine and impostor minutiae neighborhoods extracted out of 30 pairs of images. The ground truth minutiae location in these images were established manually using a semi-automated truthing tool. Half of these pairs consisted of bad prints that contained poor ridge structure and creases. The quality of the fingerprint images was established using an objective quality metric described in [11].

4.1.2. Training and Testing The training set consists of 500 impostor and genuine neighborhoods that have been normalized w.r.t orientation and brightness. The testing set consists of an equal number of impostor and genuine 


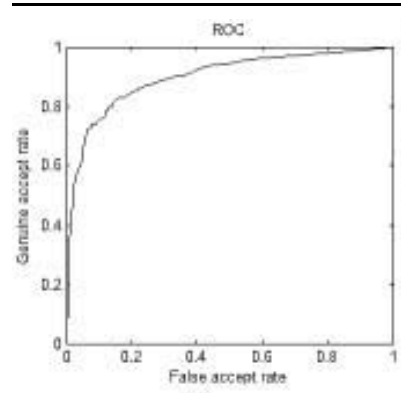

(a)

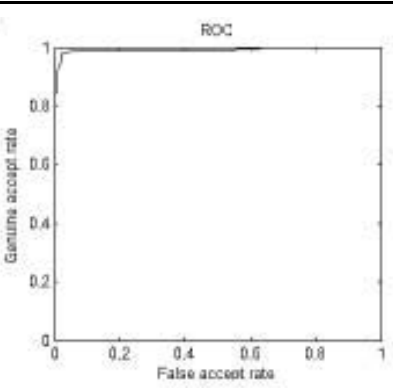

(b)
Figure 6. ROC for the (a) wedge feature classifier and (b) Gabor based classifier

neighborhoods. Ridges and bifurcation are treated uniformly since it can be emperically shown the response of the wedge filter are identical in each case. The 180 dimensional features obtained are used to train a neural network to distinguish between the two classes. The equal error rates during training and testing are $6 \%$ and $17 \%$ respectively. Figure 6 a shows the ROC curve for the wedge feature classifier.

\subsection{Gabor expansion approach}

4.2.1. Test data The gabor expansion for ridge endings and bifurcations are of opposite signs. Since we treat bifurcations as duals of the ridge endings, the negative image is used during feature extraction. The 272 features are pruned using the procedure mentioned in section 3.3. The features are classified using Bayesian classification. For sake of brevity, results using Neural networks have been omitted, since they perform less optimally than a Bayesian approach.

4.2.2. Training and testing Training uses $60 \%$ of the data and the rest is used for testing. The training results in the following pieces of information. (i)The mean vectors $\overrightarrow{\mu_{i}}$ and $\overrightarrow{\mu_{r}}$, the std. deviation vectors $\overrightarrow{\sigma_{i}}$ and $\overrightarrow{\sigma_{r}}$ corresponding to impostor and genuine neighborhoods respectively. The equal error rate during training and testing are $2 \%$ and $2 \%$ respectively. Figure $6 \mathrm{~b}$ shows the ROC curve for the Gabor expansion based classifier.

\section{Conclusion}

We have presented two novel features for minutiae verification in fingerprint images. The algorithm provides accuracy better than other gray scale approaches mentioned in literature. The approaches are computationally efficient and can also be used to design minutiae detector that can directly operate on the gray scale images. Our future work will involve fusing the decision of the two classifiers and studying the effects of minutiae verification on matching performance.

\section{References}

[1] S. Chikkerur, C. Wu, and V. Govindaraju. A systematic approach for feature extraction in fingerprint images. In International Conference on Biometric Authentication, 2004.

[2] M. D. and M. D. Neural network based minutiae filtering in fingerprint images. In 14th International Conference on Pattern Recognition, pages 1654-1658, 1998.

[3] J. Daugman. Complete discrete $2 d$ gabor transform by neural networks for image analysis and compression. Transactions on Acoustics, Speech and Signal Processing, 36:11691179, 1988.

[4] O. R. Duda, P. E. Hart., and D. G. Stork. Pattern Classification. John Wiley and Sons, NY, 2001.

[5] W. Freeman and E. Aldeman. The design and use of steerable filters. Transactions on PAMI, 13(9):891-906, 1991.

[6] L. Hong, Y. Wang, and A. K. Jain. Fingerprint image enhancement: Algorithm and performance evaluation. Transactions on PAMI, 21(4):777-789, August 1998.

[7] D. C. Hung. Enhancement and feature purification of fingerprint images. Pattern Recognition, 26(11):1661-1671, 1993.

[8] T. S. Lee. Image representation using $2 d$ gabor wavelets. Transactions on PAMI, 18(10):959-971, 1996.

[9] D. Maio and D. Maltoni. Direct gray scale minutia detection in fingerprints. Transactions on PAMI, 19(1), 1997.

[10] D. Maio, D. Maltoni, A. K. Jain, and S. Prabhakar. Handbook of Fingerprint Recognition. Springer Verlag, 2003.

[11] S. Pankanti, N. Haas, N. K. Ratha, and R. Bolle. Quantifying quality: A case study in fingerprints. In IEEE Conference on AutoID, 2002.

[12] S. Prabhakar, A. Jain, J. Wang, S. Pankanti, and R. Bolle. Minutiae verification and classification for fingerprint matching. In International Conference on Pattern Recognition, volume 1, pages 25-29, 2000.

[13] S. Qian and D. Chen. Joint Time-Frequency Analysis, Methods and Applications. Prentice Hall, 1996.

[14] D. M. R. Cappelli, D. Maio. Synthetic fingerprint-image generation. In International Conference on Pattern Recognition, 2000.

[15] M. Riedmiller and H. Braun. A direct adaptive method for faster backpropagation learning: The rprop algorithm. In International Conference on Neural Networks, 1993.

[16] E. P. Simoncelli and H. Farid. Steerable wedge filters for local orientation analysis. Transactions on Image Processing, 5(9), 1996.

[17] C. Wu, J. Zhou, Z. Bian, and G. Rong. Robust crease detection in fingerprint images. In Conference on Computer Vision and Pattern Recognition, 2003.

[18] Q. Xiao and H. Raafat. Combining Statistical and Structural Information for Fingerprint Image Processing Classifcation and Identification, pages 335-354. World Scientific, NJ, 1991. 